\title{
Measure and Helly's Intersection Theorem for Convex Sets
}

\author{
by \\ N. STAVRAKAS \\ Presented by Stanistaw KWAPIEŃ
}

In memory of Zdzisław Pawlak

\begin{abstract}
Summary. Let $\mathcal{F}=\left\{F_{\alpha}\right\}$ be a uniformly bounded collection of compact convex sets in $\mathbb{R}^{n}$. Katchalski extended Helly's theorem by proving for finite $\mathcal{F}$ that $\operatorname{dim}(\bigcap \mathcal{F}) \geq d$, $0 \leq d \leq n$, if and only if the intersection of any $f(n, d)$ elements has dimension at least $d$ where $f(n, 0)=n+1=f(n, n)$ and $f(n, d)=\max \{n+1,2 n-2 d+2\}$ for $1 \leq d \leq n-1$. An equivalent statement of Katchalski's result for finite $\mathcal{F}$ is that there exists $\delta>0$ such that the intersection of any $f(n, d)$ elements of $\mathcal{F}$ contains a $d$-dimensional ball of measure $\delta$ where $f(n, 0)=n+1=f(n, n)$ and $f(n, d)=\max \{n+1,2 n-2 d+2\}$ for $1 \leq d \leq n-1$. It is proven that this result holds if the word finite is omitted and extends a result of Breen in which $f(n, 0)=n+1=f(n, n)$ and $f(n, d)=2 n$ for $1 \leq d \leq n-1$. This is applied to give necessary and sufficient conditions for the concepts of "visibility" and "clear visibility" to coincide for continua in $\mathbb{R}^{n}$ without any local connectivity conditions.
\end{abstract}

1. Introduction. Katchalski [6] significantly generalized Helly's intersection theorem on convex sets by proving the theorem stated in the abstract. Let $\mathcal{F}=\left\{F_{\alpha}\right\}$ be a uniformly bounded collection of compact convex sets in $\mathbb{R}^{n}$. Suppose $0 \leq d \leq n, j$ is a positive integer and $\delta>0$. The collection $\mathcal{F}$ is said to have property $(j, d, \delta)$ if any $j$ elements of $\mathcal{F}$ contain a common closed $d$-dimensional ball of radius $\delta$. Breen [2] proved that if $\mathcal{F} \subseteq \mathbb{R}^{n}$ is a uniformly bounded collection of compact convex sets then $\operatorname{dim}(\bigcap \mathcal{F}) \geq d$ if and only if for some $\delta>0, \mathcal{F}$ has property $(i(n, d), d, \delta)$ where $i(n, d)=2 n, 1 \leq$ $d \leq n-1$, and $i(n, 0)=i(n, n)=n+1$. Two of the main tools she employed were Katchalski's theorem [6] and an intersection result of Falconer [5].

Our proof was in part motivated by an alternative proof for finite $\mathcal{F}$ outlined by Katchalski in [6] using the Bonnice-Klee theorem [1]. If $\mathcal{F}=$

2000 Mathematics Subject Classification: Primary 52A20.

Key words and phrases: Helly's theorem, cone, visibility. 
$\left\{F_{\alpha}\right\}$ is a uniformly bounded collection of compact convex sets in $\mathbb{R}^{n}$, then $\mathcal{F}$ is said to be $H$-closed provided $\mathcal{F}$ is closed in the sense of the Hausdorff metric. If $F \in \mathcal{F}$ the cone generated by $F$ is defined as the set $\{\lambda x \mid \lambda \geq 0$ and $x \in F\}$ and will be denoted by cone $F$; note that cone $F$ is not necessarily closed. The symbol $C(\mathcal{F})$ denotes $\{\operatorname{cone} F \mid F \in \mathcal{F}\}$. If $r>0$ the symbol $B(x, r)$ denotes the closed ball of radius $r$ with center $x$. Let $\mathcal{C}=\left\{C_{\alpha}\right\}$ be a collection of closed convex cones with apex $0_{v}$ (the origin) in $\mathbb{R}^{n}$. Suppose $1 \leq d \leq n, j$ is a positive integer, $\delta>0$, and $r>0$. The symbol $\mathcal{B}_{r}(\mathcal{C})$ denotes $\left\{B_{\alpha} \mid B_{\alpha}=C_{\alpha} \cap B\left(0_{v}, r\right), C_{\alpha} \in \mathcal{C}\right\}$. Also, $\mathcal{C}$ is said to have property $(j, d, \delta, r)$ if $\mathcal{B}_{r}(\mathcal{C})$ has property $(j, d, \delta)$. If $j$ is a positive integer and $\mathcal{F}$ is a family of sets then $\mathcal{F}^{j}$ is defined as $\{\bigcap \mathcal{A}|\mathcal{A} \subset \mathcal{F},| \mathcal{A} \mid=j\}$. Also, if $F$ is a compact convex set, $\operatorname{rad}_{j}(F)$ denotes the nonnegative number with the property that $F$ contains a closed $j$-dimensional convex ball of radius $\operatorname{rad}_{j}(F)$ and for any $\kappa>0$ the set $F$ does not contain a $j$-dimensional ball of $\operatorname{radius}_{\operatorname{rad}_{j}}(F)+\kappa$.

We shall make explicit use of the following result of Falconer [5].

Proposition 1. Let $\mathcal{F}=\left\{F_{\alpha}\right\}$ be a uniformly bounded $H$-closed collection of compact convex sets in $\mathbb{R}^{n}$. If $\operatorname{dim}(\bigcap \mathcal{F})<n$ then there exist $F_{\alpha_{1}}, \ldots, F_{\alpha_{k}}$ such that $\operatorname{dim}\left(\bigcap_{j=1}^{k} F_{\alpha_{j}}\right)=q<n$ where $k \leq 2(n-q)$.

Two linear flats $I$ and $J$ each of dimension 1 or more will be called skew if $I \cap J=\emptyset$ and whenever $I_{1} \subset I$ and $J_{1} \subset J$ are flats of dimension 1 or more then no translate $I_{1}$ is contained in $I_{2}$ and vice versa. Two convex sets $S$ and $L$ each of dimension 1 or more will be called skew if there exist two skew linear flats $I$ and $J$ with $S \subset I$ and $L \subset J$. We shall need the following proposition.

Proposition 2. Let $L \subset \mathbb{R}^{n}, n \geq 4$, be an $n$-3-dimensional subspace and let $F \subset L$ be a convex set with $0_{v} \in F$ and $1 \leq \operatorname{dim} F \leq n-3$. Let $S$ be a convex set $S$ of dimension 2 which is skew to $L$. Let $G=\operatorname{conv}(S \cup F)$ and suppose that $1 \leq m \leq n-3$. If $\operatorname{dim} F=m$ then $\operatorname{dim} G=m+3$.

Proof. Suppose $n=4$. Then $m=1$. Since $S$ is skew to $L, S$ does not intersect $L$ nor is $S$ parallel to any nontrivial flat of $L$, and $\operatorname{since} \operatorname{dim} S=2$ we have $\operatorname{dim} G \geq 3$. We claim $\operatorname{dim} G \geq 4$. Suppose not. Then $\operatorname{dim} G=3$ and if $I$ is the linear flat generated by $S$ then $\operatorname{dim} I=2$ and so either $I$ must intersect $L$ or be parallel to a flat in $L$, each of which is a contradiction. Thus $\operatorname{dim} G=4$. Thus the assertion is true for $n=4$. We now suppose that it is true for $n$ and prove it for $n+1$. If $m=1$ then $G$ is contained in a copy of $\mathbb{R}^{4}$ and the same argument as the one just given yields the assertion. Without loss of generality we may suppose that $2 \leq m \leq n-2$. Thus if $2 \leq m \leq n-3$ then $G$ is contained in a copy of $\mathbb{R}^{n}$ and the induction hypothesis gives the assertion. Thus we may suppose $m=n-2$. The same 
argument as given in the first sentence gives $\operatorname{dim} G \geq n$. If $\operatorname{dim} G=n$ then if $J$ is the $n-2$-dimensional subspace space generated by $F$ and if $I$ is the 2-dimensional linear flat generated by $S$ then in $\mathbb{R}^{n}$ either $I$ must intersect $J$ or be parallel to a flat in $J$, each of which is a contradiction. Thus $\operatorname{dim} G=n+1=(n-2)+3=m+3$ and the assertion follows.

\section{The intersection of cones and convex sets}

THEOREM 3. Let $\mathcal{F}=\left\{F_{\alpha}\right\}$ be a uniformly bounded collection of $H$ closed compact convex sets in $\mathbb{R}^{n}, n \geq 2$, with $0_{v} \in \bigcap \mathcal{F}$. Then $\operatorname{dim}(\bigcap \mathcal{F}) \geq$ $d \geq 2$ if and only if $\operatorname{dim}(\bigcap C(\mathcal{F})) \geq d \geq 2$.

Proof. The necessity is immediate; we consider the sufficiency. Let $k=$ $\operatorname{dim}(\bigcap C(\mathcal{F})) \geq 2$ and define $j=n-\operatorname{dim}(\bigcap C(\mathcal{F}))=n-k$. We first establish the assertion in the case of $j=0$, i.e. $\operatorname{dim}(\bigcap C(\mathcal{F}))=k=n$. Suppose that $\operatorname{dim}(\bigcap \mathcal{F})<n$. Then by Proposition 1 of Falconer there exist $F_{\alpha_{1}}, \ldots, F_{\alpha_{k}}$ such that $\operatorname{dim}\left(\bigcap_{j=1}^{k} F_{\alpha_{j}}\right)=q<n$. Note that $\bigcap C(\mathcal{F}) \subseteq \bigcap_{j=1}^{k} C\left(F_{\alpha_{j}}\right)=$ $C\left(\bigcap_{j=1}^{k} F_{\alpha_{j}}\right)$, which implies that $\operatorname{dim}(\bigcap C(\mathcal{F}))<\operatorname{dim}\left(\bigcap_{j=1}^{k} C\left(F_{\alpha_{j}}\right)\right)=$ $\operatorname{dim}\left(\bigcap_{j=1}^{k} F_{\alpha_{j}}\right)<n$, a contradiction. Thus the assertion is true for $d=n$, $n=2$, and we may suppose that $k<n$ and $n \geq 3$.

Let $P(j)$ be the conclusion of the theorem for $j$; the last paragraph shows that $P(0)$ is true. We now suppose that $P(j)$ is true and prove that $P(j+1)$ is true. Since $\operatorname{dim}(\bigcap C(\mathcal{F})) \geq 2$ we may choose a hyperplane $L$ with $0_{v} \in L$ such that $L$ does not support $\bigcap C(\mathcal{F})$. In particular, $L$ cannot support any element of $\mathcal{F}$, which implies by a routine argument that $L \cap \mathcal{F}$ is $H$-closed and that if $\mathcal{F}^{L}=L \cap \mathcal{F}$ then $\operatorname{dim}\left(\bigcap C\left(\mathcal{F}^{L}\right)\right)=k-1 \geq 1$. Let $V$ denote the subspace generated by $\bigcap C\left(\mathcal{F}^{L}\right)$. Regarding $\mathbb{R}^{n}$ as a subset of $\mathbb{R}^{n+1}$, since $1 \leq k-1 \leq(n+1)-3$ we may choose a compact convex set $S \subset \mathbb{R}^{n+1}$ of dimension 2 which is skew to $V$. Define $\mathcal{G}=\left\{\operatorname{conv}\left(\{S\} \cup F_{\alpha}^{L}\right) \mid F_{\alpha}^{L} \in \mathcal{F}^{L}\right\}$. Note that if $M=\operatorname{conv}\left(S \cup \bigcap C\left(\mathcal{F}^{L}\right)\right)$ then $M \subset \bigcap C(\mathcal{G})$. Since $S$ is skew to $V \subset L$ and $1 \leq k-1 \leq(n+1)-3$ we see by applying Proposition 2 in $\mathbb{R}^{n+1}$ that $\operatorname{dim} M=((k-1)+3)=k+2$ and $\operatorname{so} \operatorname{dim}(\bigcap C(\mathcal{G})) \geq \operatorname{dim} M \geq k+2$. Since

$$
(n+1)-\operatorname{dim}(\bigcap C(\mathcal{G}))=(n+1)-(k+2)=n-k-1 \leq n-k=j,
$$

the induction hypothesis on $j$ applied in $\mathbb{R}^{n+1}$ gives $\operatorname{dim}(\cap \mathcal{G}) \geq k+2$.

We next assert that $\bigcap \mathcal{G}=\operatorname{conv}\left(S \cup \cap \mathcal{F}^{L}\right)$. This follows if we show that $\bigcap \mathcal{G} \subset \operatorname{conv}\left(S \cup \cap \mathcal{F}^{L}\right)$. Let $x \in \bigcap \mathcal{G}$. If $x \in S \cup \cap \mathcal{F}^{L}$ we are done; if not then for each $F_{\alpha}^{L}, F_{\beta}^{L}$ there exist positive scalars $\lambda_{\alpha}, \lambda_{\beta}$ less than 1 and points $s_{\alpha}, s_{\beta}$ in $S, f_{\alpha} \in F_{\alpha}^{L}$ and $f_{\beta} \in F_{\beta}^{L}$ with

$$
x=\lambda_{\alpha} s_{\alpha}+\left(1-\lambda_{\alpha}\right) f_{\alpha}=\lambda_{\beta} s_{\beta}+\left(1-\lambda_{\beta}\right) f_{\beta} .
$$


Thus $\lambda_{\alpha} s_{\alpha}-\lambda_{\beta} s_{\beta}=\left(1-\lambda_{\beta}\right) f_{\beta}-\left(1-\lambda_{\alpha}\right) f_{\alpha}$. Since $S$ is skew to $V$, both $\lambda_{\alpha} s_{\alpha}-\lambda_{\beta} s_{\beta}$ and $\left(1-\lambda_{\beta}\right) f_{\beta}-\left(1-\lambda_{\alpha}\right) f_{\alpha}$ equal $0_{v}$. Note that $f_{\alpha} \neq 0_{v}$ for all $\alpha$ : if one $f_{\beta}=0_{v}$ then $f_{\alpha}=0_{v}$ for all $\alpha$ and then $x=0_{v}$ and so $x \in \bigcap \mathcal{F}^{L}$, a contradiction as $x \notin S \cup \cap \mathcal{F}^{L}$. Thus $f_{\alpha}$ and $f_{\beta}$ are positive scalar multiples of each other for any $\alpha$ and $\beta$, and as $\mathcal{F}^{L}$ is $H$-closed we may produce a set $F_{\theta}^{L} \in \mathcal{F}^{L}$ where $\left\|f_{\theta}\right\|=\inf \left\|f_{\alpha}\right\|>0$ over all $\alpha$ and $x=\lambda_{\theta} s_{\theta}+\left(1-\lambda_{\theta}\right) f_{\theta}$ with $f_{\theta} \in\left(\cap \mathcal{F}^{L}\right)$ and the assertion follows.

Let $s=\operatorname{dim}\left(\bigcap \mathcal{F}^{L}\right)$. Note that $s \geq 1$, for if $s=0$ then since $\bigcap \mathcal{G}=$ $\operatorname{conv}\left(S \cup \cap \mathcal{F}^{L}\right)$ and $\operatorname{dim} S=2$ we see that $\operatorname{dim}(\bigcap \mathcal{G})=3$, which is a contradiction as $\operatorname{dim}(\bigcap \mathcal{G}) \geq k+2 \geq 4$. Further, as $1 \leq s=\operatorname{dim}\left(\bigcap \mathcal{F}^{L}\right) \leq$ $\operatorname{dim}\left(\bigcap C\left(\mathcal{F}^{L}\right)\right)=k-1$ and $1 \leq k-1 \leq(n+1)-3$, we see by applying Proposition 2 in $\mathbb{R}^{n+1}$ that $k+2 \leq \operatorname{dim}(\bigcap \mathcal{G})=\operatorname{dim}\left(\bigcap \mathcal{F}^{L}\right)+3=s+3$ and so $s \geq d-1$ and $s=\operatorname{dim}\left(\bigcap \mathcal{F}^{L}\right) \geq k-1$. Thus we may choose a nontrivial closed line segment $h=\left[0_{v}, x\right] \subset \bigcap^{L} \subset \bigcap C(\mathcal{F})$, and as $\operatorname{dim}(\bigcap C(\mathcal{F})) \geq 2$ we may choose a hyperplane $L_{1} \neq L$ with $0_{v} \in L_{1}, h \cap L_{1}=0_{v}$ and such that $L_{1}$ does not support $\bigcap C(\mathcal{F})$. Repeating for $L_{1}$ the same construction done for $L$ gives $\operatorname{dim}\left(\cap \mathcal{F}^{L_{1}}\right) \geq k-1$. The latter together with the facts that $h \subset \bigcap C(\mathcal{F})$ and $h \cap L_{1}=0_{v}$ implies that $\operatorname{dim}(\bigcap \mathcal{F}) \geq k$, which establishes the theorem.

\section{The intersection of convex sets}

THEOREM 4. Let $\mathcal{F}=\left\{F_{\alpha}\right\}$ be a uniformly bounded collection of compact convex sets in $\mathbb{R}^{n}$. Then $\operatorname{dim}(\bigcap \mathcal{F}) \geq d, 0 \leq d \leq n$, if and only if for some $\delta>0, \mathcal{F}$ has property $(f(n, d), d, \delta)$ where $f(n, 0)=n+1$ and $f(n, d)=$ $\max \{n+2,2 n-2 d+2\}$ for $1 \leq d \leq n$.

Proof. The necessity is immediate; we consider the sufficiency. We proceed by induction on $n$. If $n \leq 2, d=1$, or $d=n$ the conclusion follows from the results of Breen [2] and Falconer [5] respectively. Thus we may suppose that $n \geq 3$ and $d \geq 2$. Without loss of generality by Helly's theorem [11] we may assume that $0_{v} \in \bigcap \mathcal{F}$. For each $F_{\alpha} \in \mathcal{F}$ let $\mathcal{H}_{\alpha}$ be the set of all closed half-spaces $H^{+}$containing $F_{\alpha}$. It is well known that $F_{\alpha}=\bigcap \mathcal{H}_{\alpha}$ [8] and therefore if $\mathcal{H}=\left\{H^{+} \mid H^{+} \in \mathcal{H}_{\alpha}, F_{\alpha} \in \mathcal{F}\right\}$ then $\bigcap \mathcal{F}=\bigcap \mathcal{H}$. As $\mathcal{F}$ has property $(f(n, d), d, \delta)$ so does $\mathcal{H}$. As $\mathcal{F}$ is uniformly bounded we may enclose the closure of $\bigcup \mathcal{F}$ in the interior of a cube $I$. Then the family $\mathcal{P}$ of polytopes which is the closure of the family $\left\{H^{+} \cap I \mid H^{+} \in \mathcal{H}\right\}$ in the Hausdorff metric, has property $(f(n, d), d, \delta), \bigcap \mathcal{F}=\bigcap \mathcal{H}, \mathcal{P}$ is $H$ closed, and each element of $C(\mathcal{P})$ is closed since it is a polytope [7]. Since $\operatorname{dim}(\bigcap \mathcal{F}) \geq \operatorname{dim}(\bigcap \mathcal{P})$ and $\bigcap \mathcal{P} \subset \bigcap \mathcal{F}$, to prove the theorem it suffices to prove $\operatorname{dim}(\bigcap \mathcal{P}) \geq d$. Therefore, without loss of generality, we suppose that $\mathcal{F}$ is an $H$-closed family of polytopes, and each element of $\mathcal{C}=C(\mathcal{F})$ is closed. By a corollary of the Bonnice-Klee theorem [1, p. 11], $\operatorname{dim}(\cap \mathcal{C}) \geq 1$. 
Recall that $\mathcal{B}=\mathcal{B}_{r}(\mathcal{C})=\left\{B_{\alpha} \mid B_{\alpha}=C_{\alpha} \cap B\left(0_{v}, r\right), C_{\alpha} \in \mathcal{C}\right\}$ and that if $r=2 \cdot \operatorname{diam}(\bigcup \mathcal{F})$ then $\mathcal{B}$ has property $(f(n, d), d, \delta)$ as does its closure $\mathcal{K}$. Since $\operatorname{dim}(\bigcap \mathcal{C})=\operatorname{dim}(\bigcap \mathcal{K})$ we see that $\operatorname{dim}(\bigcap \mathcal{K}) \geq 1$. Thus we may choose a point $u \in \operatorname{relint}(\bigcap \mathcal{K})$. We may then choose a hyperplane $H$ with $u \in H$, $\bigcap \mathcal{K} \nsubseteq H$, with $\bigcap \mathcal{K}$ intersecting both open half-spaces of $H$ and $H$ does not support $\bigcap \mathcal{K}$. Note that $\bigcap \mathcal{K} \subset E_{\beta}$ for any $E_{\beta} \in \mathcal{K}^{f(n, d)}$. Since $\bigcap \mathcal{K} \not \subset H$, each $E_{\beta} \in \mathcal{K}^{f(n, d)}$ must intersect at least one of the open half-spaces of $H$ since if not then $\bigcap \mathcal{K} \subset E_{\beta} \subset H$, a contradiction. But then $E_{\beta}$ must intersect both the open half-spaces of $H$ since if not then $H$ supports $\bigcap \mathcal{K}$, a contradiction. This together with the hypothesis that $\mathcal{K}$ has property $(f(n, d), d, \delta)$ implies that $\operatorname{dim}\left(H \cap E_{\beta}\right) \geq d-1 \geq 1$.

Suppose that $\theta_{\beta}=\operatorname{rad}_{d-1}\left(H \cap E_{\beta}\right)$ and let $\theta=\inf \left\{\theta_{\beta} \mid E_{\beta} \in \mathcal{K}^{f(n, d)}\right\}$. We next assert that $\theta>0$. Suppose that $\theta=0$. Then there exists a sequence $\left\{E_{\beta_{i}}\right\}$ in $\mathcal{K}^{f(n, d)}$ such that $\theta_{\beta_{i}} \rightarrow 0$ as $i \rightarrow \infty$ and for each $i$, $E_{\beta_{i}}=K_{\alpha\left(1, \beta_{i}\right)} \cap K_{\alpha\left(2, \beta_{i}\right)} \cap \cdots \cap K_{\alpha\left(f(n, d), \beta_{i}\right)}$. Without loss of generality (avoiding subsequences) we may suppose that $E_{\beta_{i}} \rightarrow Q$ for some compact convex set $Q$. Since for each $i, \bigcap \mathcal{K} \subset E_{\beta_{i}}$, we have $\bigcap \mathcal{K} \subset Q$ and so $Q$ must intersect both open half-spaces of $H$ since $\bigcap \mathcal{K}$ does. Further, since $\mathcal{K}$ has property $(f(n, d), d, \delta)$, each $E_{\beta_{i}}$ contains some closed $d$-dimensional ball of radius $\delta$; a standard argument in the Hausdorff metric then shows that $Q$ must contain a closed $d$-dimensional ball of radius $\delta$. Thus $\operatorname{dim} Q \geq d$. Since $Q$ must intersect both open half-spaces of $H$ we have $\operatorname{dim}(Q \cap H) \geq d-1 \geq 1$ and by a routine argument $E_{\beta_{i}} \cap H \rightarrow Q \cap H$. Since $\theta=0$ and $\theta_{\beta_{i}}=\operatorname{rad}_{d-1}\left(H \cap E_{\beta_{i}}\right)$, and $E_{\beta_{i}} \cap H \rightarrow Q$, we must have $\operatorname{dim}(Q \cap H) \leq d-2$, a contradiction. Thus $\theta=\inf _{i} \theta_{i}>0$.

Now $\theta>0$ implies that if $\mathcal{K}_{1}=\left\{H \cap K_{\alpha} \mid K_{\alpha} \in \mathcal{K}\right\}$ then $\mathcal{K}_{1}$ has property $(f(n, d), d-1, \theta)$. Since $f(n-1, d-1) \leq f(n, d), \mathcal{K}_{1}$ has property $(f(n-1, d-1), d-1, \theta)$. The induction hypothesis applied in the hyperplane $H$ yields $\operatorname{dim}\left(\bigcap \mathcal{K}_{1}\right) \geq d-1 \geq 1$. Since $\bigcap \mathcal{K}_{1}=H \cap \bigcap \mathcal{K}$ and $\bigcap \mathcal{K}$ intersects both open half-spaces of $H$, this implies that $\operatorname{dim}(\bigcap \mathcal{K}) \geq d \geq 2$. Then since $\operatorname{dim}(\bigcap \mathcal{C})=\operatorname{dim}(\bigcap \mathcal{B}) \geq \operatorname{dim}(\bigcap \mathcal{K})$ we see that $\operatorname{dim}(\bigcap \mathcal{C}) \geq d \geq 2$ and an application of Theorem 3 establishes the theorem.

4. The equivalence of visibility and clear visibility. If $S \subset \mathbb{R}^{n}$ is a nonempty set, the symbols $S(x)$, conv $S$, and Ker $S$ denote, respectively, $\{y \mid[x, y] \subset S\}$, the convex hull of $S$, and $\{x \mid[x, y] \subset S \quad \forall y \in S\}$. If $A \subset S$ and $\varepsilon>0$ let $A_{\varepsilon}$ denote all points in $S$ whose distance from $A$ is less than $\varepsilon$. If $K$ is a nonempty subset of $S$ and $x \in S$, and $\operatorname{conv}(\{x\} \cup K) \subset S$, we say that $x$ is visible via $S$ from $K$. Suppose $0 \leq d \leq n, j$ is a positive integer and $\delta>0$. $A$ is said to be $(j, d, \delta)$ visible if given a set $K$ of $j$ elements of $A$, each point of $K$ is visible via $S$ from a common $d$-dimensional ball $B_{K}$ of radius $\delta$ 
contained in $S$. Moreover, $A$ is said to be $(j, d)$ clearly visible if given a set $K$ of $j$ elements of $A$ there exists a relatively open subset $O_{K}$ of $S$ containing $K$ such that each point of $O_{K}$ is visible via $S$ from a common $d$-dimensional ball $B_{K}$ contained in $S$. Finally, $A$ is said to be $(j, d, \delta)$ clearly visible if given a set $K$ of $j$ elements of $A$ there exists a relatively open subset $O_{K}$ of $S$ containing $K$ such that each point of $O_{K}$ is visible via $S$ from a common $d$-dimensional ball $B_{K}$ of radius $\delta$ contained in $S$.

TheOrem 5. Let $S \subset \mathbb{R}^{n}$ be a nonconvex continuum. Then $S$ being $(j, d)$ clearly visible is equivalent to $S$ being $(j, d, \delta)$ visible for some $\delta>0$ if and only if $j \geq f(n, d)$ where $f(n, 0)=n+1$ and $f(n, d)=\max \{n+2,2 n-2 d+2\}$ for $1 \leq d \leq n$.

Proof. We first demonstrate the sufficiency. To prove the equivalence of the two visibility conditions it suffices to show that $S$ being $(j, d, \delta)$ visible for some $\delta>0$ implies $S$ being $(j, d)$ clearly visible since $(j, d)$ clear visibility of $S$ always implies $(j, d, \delta)$ visibility of $S$ for some $\delta>0$ (Breen [3] or Stavrakas [9, Theorem 3]). Since $j \geq f(n, d), S$ is $(f(n, d), d, \delta)$ visible. This coupled with Theorem 4 shows that $\operatorname{dim}(\bigcap\{\operatorname{conv}(S(x)) \mid x \in S\} \geq d$ and Krasnosel'skiü's lemma [11] yields $\bigcap\{\operatorname{conv}(S(x)) \mid x \in S\} \subset \operatorname{Ker} S$, which implies that $\operatorname{dim}(\operatorname{Ker} S)=d$. This immediately implies the $(j, d)$ clear visibility of $S$ for any $j \geq 1$.

To prove the necessity, it suffices to construct a continuum $S$ for which $j<f(n, d)$ such that $S$ is $(j, d, \delta)$ visible for some $\delta>0$ and $S$ is not $(j, d)$ clearly visible. To do this in $\mathbb{R}^{2}$ let $S=\bigcup_{n=1}^{\infty}\left\{\left[0_{v}, x_{n}\right] \mid x_{1}=(1,0)\right.$, $\left.x_{n}=(1,1 / n), n=2,3, \ldots\right\}$. Note that $1<f(2,1)=4, S$ is $(1,1,1 / 2)$ visible, but $0_{v}$ is not clearly visible from any one-dimensional subset of $S$ and so in particular $S$ is not $(1,1)$ clearly visible.

TheOREM 6. Let $S \subset \mathbb{R}^{n}$ be a nonconvex continuum with points of local nonconvexity $Q$. Then the following are equivalent:

(A) $S$ is $(f(n, d), d, \delta)$ visible for some $\delta>0$.

(B) $\operatorname{dim}(\operatorname{Ker} S)=d$.

(C) $S$ is $(f(n, d), d, \delta)$ clearly visible for some $\delta>0$.

(D) $Q_{\varepsilon}$ is $(f(n, d), d)$ clearly visible for some $\varepsilon>0$.

(E) $Q$ is $(f(n, d), d, \delta)$ clearly visible for some $\delta>0$.

(F) $Q_{\varepsilon}$ is $(f(n, d), d, \delta)$ visible for some $\varepsilon>0$ and $\delta>0$.

Proof. The implications $(\mathrm{B}) \Rightarrow(\mathrm{C}),(\mathrm{C}) \Rightarrow(\mathrm{D})$ and $(\mathrm{D}) \Rightarrow(\mathrm{E})$ are immediate. The implication $(\mathrm{E}) \Rightarrow(\mathrm{F})$ is established in Stavrakas [9, Theorem 3]. The implication $(\mathrm{A}) \Rightarrow(\mathrm{B})$ was established in the first paragraph of the last proof. To prove $(\mathrm{F}) \Rightarrow(\mathrm{A})$ we note that in Stavrakas [10] it is proven that $\bigcap\left\{\operatorname{conv}(S(x)) \mid x \in Q_{\varepsilon}\right\}=\bigcap\{\operatorname{conv}(S(x) \mid x \in S\}$ and so the hypothe- 
sis $(\mathrm{F})$ coupled with the same argument in the first paragraph of the proof of Theorem 5 yields the conclusion (B), which immediately implies (A).

We remark that examples given in Breen [3] illustrate for each $d \geq 1$ that the number $f(n, d)$ is best possible in the sense that the above theorem fails if $f(n, d)$ is replaced by a smaller integer. We remark that J. Cel [4] has used a generalized form of Krasnosel'skiı̌'s lemma to represent starshaped sets in normed linear spaces.

In conclusion we acknowledge the many and profound accomplishments, in convexity and infinite-dimensional topology, of Victor Klee who passed away this year.

\section{References}

[1] W. Bonnice and V. Klee, The generation of convex hulls, Math. Ann. 152 (1963), $1-29$.

[2] M. Breen, Krasnosel'skii numbers for bounded finitely starlike sets in $R^{d}$, J. Geom. 37 (1990), 48-54.

[3] -, Improved Krasnosel'skii theorems for the dimension of the kernel of a starshaped set, ibid. 27 (1986), 175-179.

[4] J. Cel, Representations of starshaped sets in normed linear spaces, J. Funct. Anal. 174 (2000) 264-275.

[5] K. J. Falconer, The dimension of the kernel of a compact starshaped set, Bull. London Math. Soc. 9 (1977), 313-316.

[6] M. Katchalski, The dimensions of the intersections of convex sets, Israel J. Math. 10 (1971), 465-470.

[7] V. Klee, Some characterizations of convex polyhedra, Acta Math. 100 (1959), 79-107.

[8] H. H. Schaefer, Topological Vector Spaces, Macmillan, New York, 1966.

[9] N. Stavrakas, Reflexivity and visibility, Bull. Polish Acad. Sci. Math. 41 (1993), 113-121.

[10] -, A reduction theorem for the intersection of closed convex hulls, Houston J. Math. 17 (1991), 271-277.

[11] F. Valentine, Convex Sets, McGraw-Hill, New York, 1964.

N. Stavrakas

Department of Mathematics

University of North Carolina

Charlotte, NC 28223, U.S.A.

E-mail: nstavrks@uncc.edu

Received September 3, 2006;

received in final form April 11, 2008 